\title{
SOME REMARKS ABOUT SERIES
}

\section{Tadeusz Janaszak}

\begin{abstract}
This paper presents a proof of the classical theorem of the theory of series. This proof would be used in lectures on the series theory.
\end{abstract}

Keywords: series, convergent, divergent series, harmonic series.

Jel Classification: C00.

DOI: $10.15611 / \mathrm{dm} .2016 .13 .03$.

\section{Introduction}

In the lectures of calculus the direct proof of the theorem that the series $\sum_{n=1}^{\infty} \frac{1}{n^{\alpha}}$ is convergent for $1<\alpha$ is rarely introduced. The knowledge about the convergence of this series is used in exercises but the proof of the convergence of this series is presented on the whole by the integral criterion. In standard textbooks of calculus it is difficult to find a direct proof of convergence of the series. This paper presents a direct proof of convergence of the series. This text is a supplement for numerous books of calculus.

Theorem. The series

$$
\sum_{n=1}^{\infty} \frac{1}{n^{\alpha}}
$$

is divergence where $0<\alpha \leq 1$ and convergence where $1<\alpha$.

For the proof of the theorem it is necessary to show a lot of lemmata.

Lemma 1. The harmonic series $\sum_{n=1}^{\infty} \frac{1}{n}$ is divergent. 
Proof. The harmonic series is equal such that:

$$
\sum_{n=1}^{\infty} \frac{1}{n}=\frac{1}{1}+\frac{1}{2}+\frac{1}{3}+\frac{1}{4}+\frac{1}{5}+\ldots+\frac{1}{8}+\ldots+\frac{1}{2^{n}+1}+\ldots+\frac{1}{2^{n+1}}+\ldots .
$$

It is possible to group the terms of harmonic series:

$$
\begin{gathered}
a_{0}=1+\frac{1}{2^{1}}=\frac{3}{2}, a_{1}=\frac{1}{2^{1}+1}+\frac{1}{2^{2}}=\frac{1}{3}+\frac{1}{4}, \\
a_{2}=\frac{1}{2^{2}+1}+\frac{1}{2^{2}+2}+\frac{1}{2^{2}+3}+\frac{1}{2^{3}}=\frac{1}{5}+\frac{1}{6}+\frac{1}{7}+\frac{1}{8}, \ldots, \\
a_{n}=\frac{1}{2^{n}+1}+\ldots+\frac{1}{2^{n+1}} .
\end{gathered}
$$

The harmonic series is equal

$$
\sum_{n=1}^{\infty} \frac{1}{n}=\sum_{n=0}^{\infty} a_{n}
$$

It is obvious that $1+\frac{1}{2}>\frac{1}{2}$, and $\frac{1}{3}+\frac{1}{4}>\frac{1}{4}+\frac{1}{4}=\frac{1}{2}$, and $\frac{1}{5}+\ldots+\frac{1}{8}>\frac{1}{8}+\ldots+\frac{1}{8}=\frac{1}{2}$, and $\frac{1}{2^{n}+1}+\ldots+\frac{1}{2^{n}+2^{n}}>2^{n} \cdot \frac{1}{2^{n+1}}=\frac{1}{2}$. From here the result below is true:

$$
\sum_{n=1}^{\infty} \frac{1}{n}>\frac{1}{2}+\frac{1}{2}+\ldots
$$

i.e. the harmonic series $\sum_{n=1}^{\infty} \frac{1}{n}$ is divergent.

Proof of the theorem: the series $\sum_{n=1}^{\infty} \frac{1}{n^{\alpha}}$ is divergence for $0<\alpha \leq 1$.

If $\alpha$ is a number such that $0<\alpha<1$ then for natural numbers the inequality $n^{\alpha}<n$ holds, hence the unequal $\frac{1}{n}<\frac{1}{n^{\alpha}}$ holds too, i.e. the harmonic series $\sum_{n=1}^{\infty} \frac{1}{n}$ is a minorant of series $\sum_{n=1}^{\infty} \frac{1}{n^{\alpha}}$ for $0<\alpha<1$. Hence the minorant is a divergence series the series (1) is divergence for $0<\alpha<1$. 
Lemma 2. For each natural number $n$ the expression is true:

$$
\frac{1}{1 \cdot 2}+\frac{1}{2 \cdot 3}+\ldots+\frac{1}{n \cdot(n+1)}=\frac{n}{n+1} .
$$

Proof by induction.

For $n=1$ the left side is equal $\frac{1}{2}$ and the right side is equal $\frac{1}{2}$ too. Suppose that for some $n$ the expression above holds. It is necessary to prove that

$$
\frac{1}{1 \cdot 2}+\frac{1}{2 \cdot 3}+\ldots+\frac{1}{n \cdot(n+1)}+\frac{1}{(n+1)(n+2)}=\frac{n+1}{n+2} .
$$

The left side of the equality above by the induction assumption is equal

$$
\frac{n}{n+1}+\frac{1}{(n+1)(n+2)}=\frac{n \cdot(n+2)+1}{(n+1)(n+2)}=\frac{n^{2}+2 n+1}{(n+1)(n+2)}=\frac{(n+1)^{2}}{(n+1)(n+2)},
$$

it is obvious that

$$
\frac{(n+1)^{2}}{(n+1)(n+2)}=\frac{n+1}{n+2}
$$

so the proof of the lemma is complete.

Corollary. The series $\sum_{n=1}^{\infty} \frac{1}{n \cdot(n+1)}$ is convergent, the sum is equal to 1 .

Proof. Because $\sum_{n=1}^{\infty} \frac{1}{n \cdot(n+1)}=\lim _{n \rightarrow \infty}\left(\sum_{k=1}^{n} \frac{1}{n \cdot(n+1)}\right)=\lim _{n \rightarrow \infty} \frac{n}{n+1}=1$, the thesis of the corollary is true.

Lemma 3. The series $\sum_{n=1}^{\infty} \frac{1}{n^{2}}$ is convergent and the sum of this is less than or equal to 2 .

$$
\sum_{n=1}^{\infty} \frac{1}{n^{2}}=1+\frac{1}{2 \cdot 2}+\frac{1}{3 \cdot 3}+\frac{1}{4 \cdot 4}+\ldots \leq 1+\frac{1}{1 \cdot 2}+\frac{1}{2 \cdot 3}+\frac{1}{3 \cdot 4}+\ldots
$$


Proof. By this expression the conclusion below holds:

$$
\sum_{n=1}^{\infty} \frac{1}{n^{2}} \leq 1+\sum_{n=1}^{\infty} \frac{1}{n \cdot(n+1)}=2 \text {. }
$$

The lemma is true.

Lemma 4. The series $\sum_{n=1}^{\infty} \frac{1}{n^{\alpha}}$ is convergent for $\alpha=1+\frac{1}{2}$.

Proof. It is necessary to see the expressions:

$$
\begin{gathered}
\sum_{n=1}^{\infty} \frac{1}{n \cdot \sqrt{n}}=\frac{1}{1 \cdot \sqrt{1}}+\frac{1}{2 \cdot \sqrt{2}}+\frac{1}{3 \cdot \sqrt{3}}+\frac{1}{4 \cdot 2}+\frac{1}{5 \cdot \sqrt{5}}+\ldots+\frac{1}{8 \sqrt{8}}+ \\
+\frac{1}{9 \cdot 3}+\frac{1}{10 \cdot \sqrt{10}}+\ldots+\frac{1}{15 \cdot \sqrt{15}}+\frac{1}{16 \cdot 4}+\frac{1}{17 \cdot \sqrt{17}}+\ldots+\frac{1}{24 \cdot \sqrt{24}}+\ldots+ \\
+\frac{1}{k^{2} \cdot k}+\frac{1}{\left(k^{2}+1\right) \cdot \sqrt{k^{2}+1}}+\ldots+\frac{1}{\left(k^{2}+2 k\right) \cdot \sqrt{k^{2}+2 k}}+\ldots
\end{gathered}
$$

i.e.

$$
\sum_{n=1}^{\infty} \frac{1}{n \cdot \sqrt{n}}=\sum_{k=1}^{\infty}\left(\sum_{j=0}^{2 k} \frac{1}{\left(k^{2}+j\right) \cdot \sqrt{k^{2}+j}}\right) .
$$

It is possible to write some obvious inequalities

$$
\frac{1}{1 \sqrt{1}}+\frac{1}{2 \cdot \sqrt{2}}+\frac{1}{3 \cdot \sqrt{3}} \leq \frac{1}{1}+\frac{1}{1}+\frac{1}{1}=\frac{2 \cdot 1+1}{1^{3}}=3,
$$

and

$$
\frac{1}{4 \cdot 2}+\frac{1}{5 \cdot \sqrt{5}}+\ldots+\frac{1}{8 \cdot \sqrt{8}} \leq \frac{1}{4 \cdot 2}+\frac{1}{4 \cdot 2}+\ldots+\frac{1}{4 \cdot 2}=\frac{2 \cdot 2+1}{2^{3}}=\frac{5}{8},
$$

and

$$
\frac{1}{9 \cdot 3}+\frac{1}{10 \cdot \sqrt{10}}+\ldots+\frac{1}{15 \cdot \sqrt{15}} \leq \frac{1}{9 \cdot 3}+\frac{1}{9 \cdot 3}+\ldots+\frac{1}{9 \cdot 3}=\frac{2 \cdot 3+1}{3^{3}}=\frac{7}{27},
$$

and

$$
\frac{1}{16 \cdot 4}+\frac{1}{17 \cdot \sqrt{17}}+\ldots+\frac{1}{24 \cdot \sqrt{24}} \leq \frac{1}{16 \cdot 4}+\frac{1}{16 \cdot 4}+\ldots+\frac{1}{16 \cdot 4}=\frac{2 \cdot 4+1}{4^{3}}=\frac{9}{64},
$$


and generally

$$
\begin{aligned}
\frac{1}{k^{2} \cdot k} & +\frac{1}{\left(k^{2}+1\right) \cdot \sqrt{k^{2}+1}}+\ldots+\frac{1}{\left(k^{2}+2 k\right) \cdot \sqrt{k^{2}+2 k}} \\
& \leq \frac{1}{k^{2} \cdot k}+\frac{1}{k^{2} \cdot k}+\ldots+\frac{1}{k^{2} \cdot k}=\frac{2 k+1}{k^{3}} .
\end{aligned}
$$

By this the inequality

$$
\sum_{k=1}^{\infty}\left(\sum_{j=0}^{2 k} \frac{1}{\left(k^{2}+j\right) \cdot \sqrt{k^{2}+j}}\right) \leq \sum_{k=1}^{\infty} \frac{2 k+1}{k^{3}}
$$

holds i.e. the evaluation

$$
\sum_{n=1}^{\infty} \frac{1}{n \cdot \sqrt{n}} \leq \sum_{k=1}^{\infty} \frac{2 k+1}{k^{3}}
$$

is true. For every natural number $k$ the expression

$$
\frac{2 k+1}{k^{3}} \leq \frac{3 k}{k^{3}}=\frac{3}{k^{2}}
$$

holds, so the series $\sum_{n=1}^{\infty} \frac{4}{n^{2}}=3 \cdot \sum_{n=1}^{\infty} \frac{1}{n^{2}}$ is a majorant of the series $\sum_{n=1}^{\infty} \frac{1}{n \cdot \sqrt{n}}$ i.e.

$$
\sum_{n=1}^{\infty} \frac{1}{n \cdot \sqrt{n}} \leq 3 \cdot \sum_{n=1}^{\infty} \frac{1}{n^{2}}
$$

Because the series $\sum_{n=1}^{\infty} \frac{1}{n^{2}}$ is convergent, by lemma 3, so the series $\sum_{n=1}^{\infty} \frac{1}{n \cdot \sqrt{n}}$ is convergent too. The sum of the series is less than or equal to 6 .

Lemma 5. If the series $\sum_{n=1}^{\infty} \frac{1}{n^{\alpha}}$ is convergent for $\alpha=1+\frac{1}{2^{s}}$ where $s$ is some natural number, then it is convergent for $\alpha=1+\frac{1}{2^{s+1}}$ too. 
Proof. Let for $s \in N$ to be $\beta(s)=\frac{1}{2^{s}}$. It is necessary to show that the series $\sum_{n=1}^{\infty} \frac{1}{n \cdot n^{\beta(s+1)}}$ is convergent if the series $\sum_{n=1}^{\infty} \frac{1}{n \cdot n^{\beta(s)}}$ is convergent. The series $\sum_{n=1}^{\infty} \frac{1}{n \cdot n^{\beta(s+1)}}$ is equal to $\sum_{n=1}^{\infty} \frac{1}{n \cdot \sqrt{n^{\beta(s)}}}$ i.e.

$$
\sum_{n=1}^{\infty} \frac{1}{n \cdot n^{\beta(s+1)}}=\frac{1}{1 \cdot \sqrt{1^{\beta(s)}}}+\frac{1}{2 \cdot \sqrt{2^{\beta(s)}}}+\frac{1}{3 \cdot \sqrt{3^{\beta(s)}}}+\ldots
$$

The inequalities below obviously hold:

$$
\frac{1}{1 \cdot \sqrt{1^{\beta(s)}}}+\frac{1}{2 \cdot \sqrt{2^{\beta(s)}}}+\frac{1}{3 \cdot \sqrt{3^{\beta(s)}}} \leq \frac{1}{1}+\frac{1}{1}+\frac{1}{1}=\frac{2 \cdot 1+1}{1}=3
$$

and

$$
\frac{1}{4 \cdot 2^{\beta(s)}}+\frac{1}{5 \cdot \sqrt{5^{\beta(s)}}}+\ldots+\frac{1}{8 \cdot \sqrt{8^{\beta(s)}}} \leq \frac{5}{4 \cdot 2^{\beta(s)}}
$$

and

$$
\frac{1}{9 \cdot 3^{\beta(s)}}+\frac{1}{10 \cdot \sqrt{10^{\beta(s)}}}+\ldots+\frac{1}{15 \cdot \sqrt{15^{\beta(s)}}} \leq \frac{7}{8 \cdot 3^{\beta[s]}}
$$

and

$$
\frac{1}{16 \cdot 4^{\beta(s)}}+\frac{1}{17 \cdot \sqrt{17^{\beta(s)}}}+\ldots+\frac{1}{24 \cdot \sqrt{24^{\beta(s)}}} \leq \frac{9}{16 \cdot 4^{\beta(s)}}
$$

and generally

$$
\frac{1}{k^{2} \cdot k^{\beta(s)}}+\frac{1}{\left(k^{2}+1\right) \cdot \sqrt{\left(k^{2}+1\right)^{\beta(s)}}}+\ldots+\frac{1}{\left(k^{2}+2 k\right) \cdot \sqrt{\left(k^{2}+2 k\right)^{\beta(s)}}} \leq \frac{2 k+1}{k^{2} \cdot k^{\beta(s)}} .
$$

The equality below is certain:

$$
\frac{2 k+1}{k^{2} \cdot k^{\beta(s)}} \leq \frac{3 k}{k^{2} \cdot k^{\beta(s)}}=\frac{3}{k \cdot k^{\beta(s)}},
$$

by this it is obvious that

$$
\sum_{n=1}^{\infty} \frac{1}{n \cdot n^{\beta(s+1)}} \leq 3 \cdot \sum_{n=1}^{\infty} \frac{1}{n \cdot n^{\beta(s)}},
$$


by the comparison test for convergence of infinite series the series $\sum_{n=1}^{\infty} \frac{1}{n \cdot n^{\beta(s+1)}}$ is convergent if the series $\sum_{n=1}^{\infty} \frac{1}{n \cdot n^{\beta(s)}}$ is convergent. The sum of the series $\sum_{n=1}^{\infty} \frac{1}{n \cdot n^{\beta(s+1)}}$ is less than or equal to $2 \cdot 3^{s+1}$. By mathematical induction there is the finish of lemma 5.

Proof of the theorem: the series $\sum_{n=1}^{\infty} \frac{1}{n^{\alpha}}$ is convergence where $1<\alpha$. If $1<\alpha$ that there is a natural number $s$ such that $1+\frac{1}{2^{s}}<\alpha$ i.e. $1+\beta[s]<\alpha$ so for each natural $n$ it is $n^{1+\beta(s)}<n^{\alpha}$ and consequently

$$
\frac{1}{n^{\alpha}}<\frac{1}{n^{1+\beta(s)}},
$$

by this the series $\sum_{n=1}^{\infty} \frac{1}{n \cdot n^{\beta(s)}}$ is a majorant of the series $\sum_{n=1}^{\infty} \frac{1}{n^{\alpha}}$ and consequently the series $\sum_{n=1}^{\infty} \frac{1}{n^{\alpha}}$ is convergent. The proof of the theorem is finished.

Usually the proof of the theorem is shown by the integral test for convergence:

On the interval $[m, \infty)$ where $m \in N$ the function $f(x)$ is positive and decreasing then the series $\sum_{n=m}^{\infty} f(n)$ and the integral $\int_{m}^{\infty} f(x) d x$ are both at the same time convergent or divergent.

Proof of the theorem with use the integral test for convergence.

The integral $\int_{1}^{\infty} \frac{1}{x} d x$ is divergence because $\lim _{t \rightarrow \infty} f(t)=\infty$. For $\alpha \neq 1$ the indefinite integral is equal: $\int \frac{1}{x^{\alpha}}=\frac{1}{1-\alpha} \cdot x^{1-\alpha}$. The value of the antiderivative at the point $x=1$ is equal $\frac{1}{\alpha-1}$, the limit $\lim _{x \rightarrow \infty} x^{1-\alpha}$ is equal to zero for 
$1<\alpha$ and it is infinity for $0<\alpha<1$. So $\int_{1}^{\infty} \frac{1}{x^{\alpha}} d x$ equals $\frac{1}{\alpha-1}$ for $1<\alpha$ and infinity for $0<\alpha<1$. This conclusion finishes the proof of the theorem.

The direct proof of the convergence of the series $\sum_{n=1}^{\infty} \frac{1}{n^{\alpha}}$ for $1<\alpha$ in another way is presented in [Fihtenholz 1978, vol. 2, p. 227], the proof of the divergence is presented in the same way as in this paper.

\section{Bibliography}

Antoniewicz R., Misztal A. (2000). Matematyka dla studentów ekonomii. PWN. Warszawa. Fichtenholz G.M. (1978). Rachunek różniczkowy i całkowy. PWN. Warszawa.

Gewert M., Skoczylas Z. (2005). Analiza matematyczna 2. Oficyna Wydawnicza GIS. Wrocław.

Smoluk A. (2007). Podstawy analizy matematycznej. Wydawnictwo Akademii Ekonomicznej we Wrocławiu. 